\title{
Low-risk gestational trophoblastic neoplasia: a single-center experience from Saudi Arabia
}

\author{
Abdulaziz Alobaid, Samer Ahmeed, Mohammed Abuzaid, Latifa Aldakhil'1, Ahmed Abu-Zaid²,3 \\ Department of Obstetrics and Gynecology, King Fahad Medical City, ${ }^{1}$ Department of Obstetrics and Gynecology, King Khaled University \\ Hospital, ${ }^{2}$ College of Medicine, Alfaisal University, Riyadh, Kingdom of Saudi Arabia, ${ }^{3}$ College of Graduate Health Sciences, University of \\ Tennessee Health Science Center, Memphis, Tennessee, USA
}

\begin{tabular}{|c|}
\hline Access this article online \\
\hline Website: www.avicennajmed.com \\
\hline DOI: 10.4103/ajm.AJM_188_18 \\
\hline Quick Response Code: \\
\hline \\
\hline
\end{tabular}

\begin{abstract}
Objective: To report our single-center experience in terms of patient clinical characteristics, treatment outcomes, and chemotherapy-related toxicities in patients with low-risk gestational trophoblastic neoplasia (GTN). Materials and Methods: A retrospective cross-sectional study (2008-2013) was conducted at a tertiary health-care hospital in Saudi Arabia. Forty-four $(n=44)$ patients met the inclusion criteria for low-risk GTN. Methotrexate (MTX) was administered in a 5-day regimen: $0.3-0.5 \mathrm{mg} / \mathrm{kg}$ intravenously (IV) daily for 5 days every 2 weeks (maximum $25 \mathrm{mg}$ per dose). Actinomycin D (ActD) was administered $1.25 \mathrm{mg} / \mathrm{m}^{2}$ pulsed IV every 2 weeks. Results: The majority of patients had molar pregnancy as the antecedent event $(86 \%)$, developed GTN within the first 4 months after the initial evacuation (93.2\%), had human chorionic gonadotropin levels between 1,000 and $10,000 \mathrm{mlU} / \mathrm{dL}(36.3 \%)$, and had the World Health Organization prognostic scores from 0 to $2(48.7 \%)$. Only 38 patients accepted treatment with chemotherapy. A total of 37 patients received first-line MTX; 34 patients of them achieved complete remission (CR, 92\%). The three patients who developed MTX resistance were salvaged with sequential ActD and all achieved CR of $100 \%$. Only one patient received first-line ActD and achieved CR. The overall survival as well as cure rate for all patients with low-risk GTN was $100 \%$. No patient developed MTX-related hepatic toxicity or ActD-related blister formation. No severe adverse effects occurred. Conclusion: Our 5-day IV MTX regimen was highly effective in treating patients with low-risk GTN, with CR rate of $92 \%$ and no severe toxicity. Primary and sequential ActD therapy appears to be very effective.
\end{abstract}

Key words: Actinomycin D, gestational trophoblastic neoplasia, methotrexate, Saudi Arabia

\section{INTRODUCTION}

Gestational trophoblastic neoplasia (GTN) refers to a cluster of biologically malignant tumors that are composed of abnormal proliferation of trophoblastic tissue. GTN comprises four categories, namely: invasive mole, choriocarcinoma, placental site trophoblastic tumor, and epithelioid trophoblastic tumor. ${ }^{[1]}$ GTN most often takes place after molar pregnancy, but it can also occur after non-molar pregnancy (for example, miscarriage and term pregnancy). ${ }^{[2]}$ GTN is greatly sensitive to chemotherapy with curable rates surpassing $90 \% .^{[1,3,4]}$

Address for correspondence: Dr. Ahmed Abu-Zaid, College of Medicine, Alfaisal University, Riyadh 11533, Kingdom of Saudi Arabia.

E-mail: aabuzaid@live.com
GTN is categorized into low- and high-risk disease. The classification is based on the International Federation of Gynecology and Obstetrics (FIGO) anatomic staging and the modified World Health Organization (WHO) prognostic risk score. This joint classification largely predicts the likelihood of disease progression and resistance to single-agent chemotherapy. ${ }^{[5]}$ Almost all patients with

This is an open access journal, and articles are distributed under the terms of the Creative Commons Attribution-NonCommercial-ShareAlike 4.0 License, which allows others to remix, tweak, and build upon the work non-commercially, as long as appropriate credit is given and the new creations are licensed under the identical terms.

For reprints contact: reprints@ @medknow.com

Cite this article as: Alobaid A, Ahmeed S, Abuzaid M, Aldakhil L, Abu-Zaid A. Low-risk gestational trophoblastic neoplasia: A single-center experience from Saudi Arabia. Avicenna J Med 2019;9:89-93. 
low-risk GTN are cured with single-agent chemotherapy, resulting in survival rates approaching nearly $100 \%$. The most frequently used single-agent chemotherapy regimens include methotrexate (MTX) \pm folinic acid (FA), or actinomycin $\mathrm{D}(\mathrm{ActD}){ }^{[6]}$

Globally, up to the present time, there is no universally agreed upon consensus concerning the optimal single-agent regimen and dosing in patients with low-risk GTN. ${ }^{[6]}$ Furthermore, locally in the context of Saudi Arabia, to the best of our knowledge, only two studies have so far reported their experiences in the management of patients with low-risk GTN. ${ }^{[2,7]}$

This study has two endpoints. The primary endpoint is to report our single-center experience in terms of patient clinical characteristics, treatment outcomes, and chemotherapy-related toxicities in patients with low-risk GTN. The secondary endpoint is to briefly compare and contrast our findings to the two published experiences in Saudi Arabia, ${ }^{[2,7]}$ and shed light on the conclusions drawn from a recently published network meta-analysis study on the topic. ${ }^{[6]}$

\section{MATERIALS AND METHODS}

The study was conducted at the Women's Specialized Hospital, King Fahad Medical City (KFMC), Riyadh, Saudi Arabia. KFMC is a tertiary health-care hospital, and the total number of deliveries ranges from 3900 to 5200 per year. The study protocol was approved by the Institutional Review Board.

From January 2008 to December 2013, the medical records of all patients diagnosed with low-risk GTN were retrospectively analyzed for clinical and treatment details. The diagnosis of GTN was established by clinical and/or histopathological criteria. Clinically, GTN was diagnosed after molar or non-molar pregnancy when there was (i) a plateau $(<15 \%$ drop) in the level of human chorionic gonadotropin (hCG) in four readings during 3 weeks, (ii) a $10 \%$ increase in hCG level for three readings during 2 weeks, or (iii) an elevation in hCG level at 6 months after molar evacuation. ${ }^{[2]}$

After GTN diagnosis was made, oncology staging workup was performed (according to hospital protocol), which included history taking, physical examination, laboratory testing, and imaging. With regard to imaging, chest X-ray with computed tomography (CT) scan of the chest, abdomen, and pelvis were routinely performed. Furthermore, CT scan or magnetic resonance imaging of the brain was performed, only if the other radiographic modalities (i.e., X-ray and CT scan) showed findings of metastatic disease. Afterward, patients were categorized into either low- or high-risk disease. This categorization was based on the FIGO anatomic staging and the WHO prognostic risk score. ${ }^{[5]}$ The WHO prognostic risk score was determined by age, antecedent pregnancy, interval from antecedent pregnancy to start of chemotherapy, pretreatment serum hCG level, largest tumor size, site of metastases, number of metastases, and prior failed chemotherapy. Low-risk disease was determined by one of the following conditions: (i) FIGO stage I GTN or (ii) stage II or III GTN with a WHO risk score less than 7. On the contrary, high-risk disease was determined by one of the following conditions: (i) FIGO stage IV disease or (ii) FIGO Stage II or III GTN with a WHO risk score more than 6.

The study inclusion criteria included only patients with lowrisk GTN. Patients were analyzed for clinical characteristics, chemotherapy regimen, toxicity profile, response rate, and 1 -year survival. The chemotherapy-related side effects were assessed in accordance with the Common Terminology Criteria for Adverse Events (CTCAE), version 5.0 (https:// ctep.cancer.gov/protocolDevelopment/electronic applications/docs/CTCAE_v5_Quick_Reference_8.5x11. pdf). The study exclusion criteria included patients with high-risk GTN or missing parameters of the WHO prognostic risk score.

At our hospital, the first-line chemotherapy for patients with low-risk GTN is a 5-day MTX regimen: $0.3-0.5 \mathrm{mg} /$ $\mathrm{kg}$, intravenously (IV) daily for 5 days and repeated every 2 weeks (maximum, $25 \mathrm{mg}$ per dose). Patients who are resistant to MTX will be salvaged with pulsed IV ActD $1.25 \mathrm{mg} / \mathrm{m}^{2}$ every 2 weeks. Complete remission (CR) was established when three consecutive weekly hCG levels were within the reference range $(<5 \mathrm{mIU} / \mathrm{dL})$. Two additional schedules of chemotherapy of the last effective dose were often administered after the first normal hCG level as a consolidation therapy.

After CR was achieved, all patients were followed up in the clinic for 12 months with monthly hCG level to assess for recurrence. Oral contraceptive use was the primary method of contraception during the treatment and follow-up periods. Hysterectomy was used as a method of evacuation if no further pregnancies were desired. Disease relapse was established when the hCG level rose after an initial CR. Disease progression was established when hCG level increased or reached a plateau in two consecutive hCG values over a 2 -week interval ${ }^{[8]}$ or when new metastases were detected. ${ }^{[9]}$ 
Data were entered into Microsoft Excel (Microsoft, Redmond, WA) for analysis. Qualitative data were reported as numbers and percentages. Quantitative data were reported as medians and ranges. Two-tailed Mann-Whitney $U$ test and chi-square tests were used for univariate analyses of continuous and categorical data, respectively. Survival analysis of the overall survival (OS) was calculated according to the Kaplan-Meier estimates method and compared by using two-tailed log-rank test. All statistical analyses were performed using the Statistical Package for the Social Sciences (SPSS) software, version 22, for Windows (IBM, Armonk, NY). For all purposes, $P$ values $<0.05$ were regarded as statistically significant.

\section{RESULTS}

A total of 44 patients met the study inclusion criteria of low-risk GTN. Over the 6 years, from 2008 to 2013, the estimated number of deliveries was 33,000. Therefore, the estimated prevalence of low-risk GTN at the study center was 1.33 per 1000 deliveries.

Table 1 shows the patient clinical characteristics $(n=44)$. The median age was 36.5 years (range, $20-52$ years). The majority of patients had molar pregnancy as the antecedent event (86\%), developed GTN within the first 4 months after the initial evacuation (93.2\%), had hCG levels between 1,000 and $10,000 \mathrm{mIU} / \mathrm{dL}$ (36.3\%), had FIGO stage I (90.9\%), and had WHO prognostic scores from 0 to $2(48.7 \%)$. Only 38 patients accepted chemotherapy treatment, as follows: 37 and 1 patient(s) received MTX and ActD as the primary single-agent chemotherapy, respectively. The reason for ActD administration in the single patient was due to a MTX contraindication for hepatic dysfunction; the patient had an uneventful outcome and achieved CR. The clinical outcomes of the six patients $(n=6)$ who refused chemotherapy were not known, and the patients were excluded from the survival analysis.

Table 2 shows the treatment outcomes for patients who received MTX as the primary single-agent chemotherapy $(n=37)$. There were 34 complete responses $(92 \%)$ and 3 treatment failures (8\%). With respect to the median age, no statistically significant difference was observed between patients with complete and failed response $(P>$ 0.05, two-tailed Mann-Whitney U test). The median WHO prognostic score for patients with failed MTX treatment was 5 (range, 3-5), whereas the score was 2 (range, $0-6$ ) for those who responded completely. The three patients who failed primary MTX therapy were treated with sequential singleagent ActD chemotherapy and all achieved CR. The OS as well as cure rate for all patients with low-risk GTN was $100 \%$.

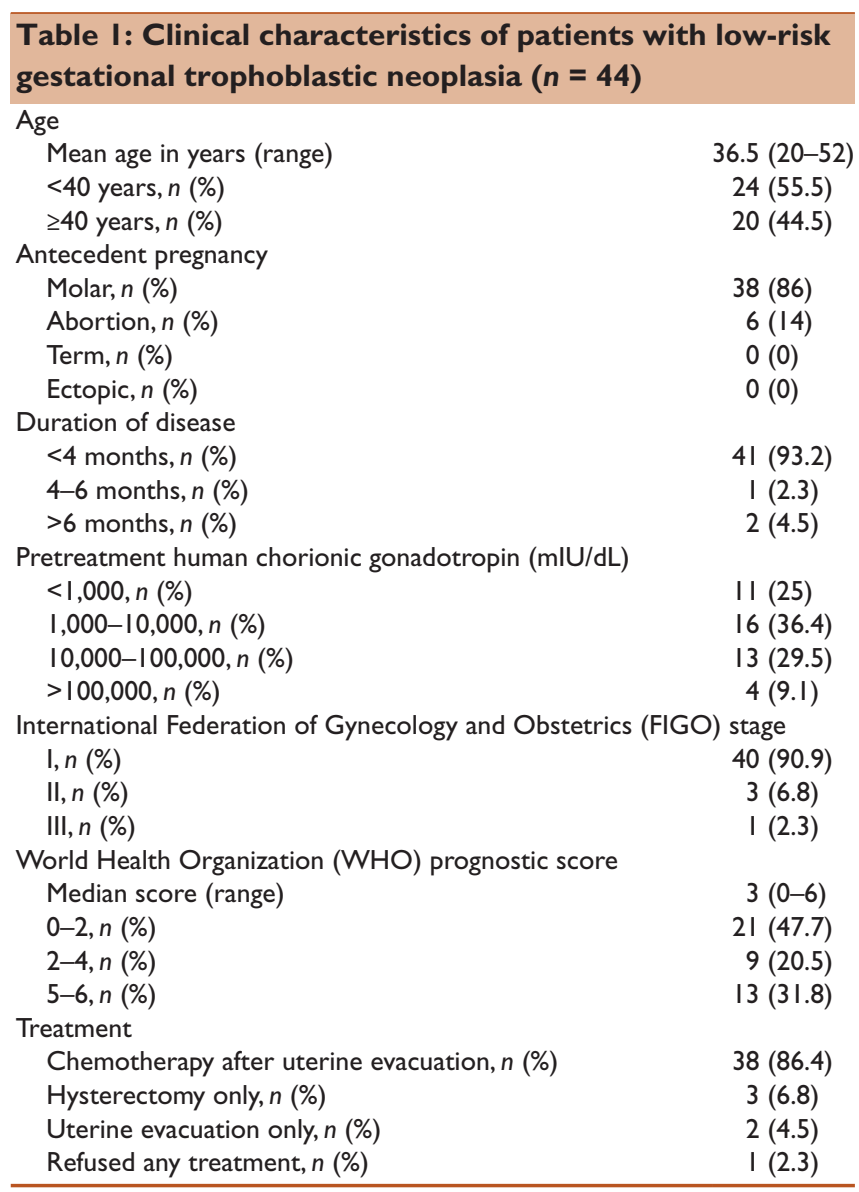

With respect to chemotherapy toxicity profile, no patient developed MTX-related hepatic toxicity or ActD-related blister formation. Among the patients who received primary MTX therapy $(n=37)$, three patients developed grade-I oral mucositis and two patients developed grade-II bone marrow suppression. Conversely, among the patients who received primary $(n=1)$ and sequential ActD therapy $(n=3)$, only one patient developed grade-II oral mucositis and another one patient developed grade-III bone marrow suppression.

\section{DISCUSSION}

Generally, the prognosis of patients with primary or recurrent (resistant) low-risk GTN is very favorable; the OS rate with chemotherapy treatment can approach $90 \%-100 \%{ }^{[2,7,10,11]}$ Similarly, in our study, primary and sequential single-agent chemotherapy treatment was collectively associated with an OS rate of $100 \%$. A 1-year OS was the main survival endpoint in this particular study; however, long-term survival data will be reported in a forthcoming communication.

Low-risk GTN is extremely sensitive to chemotherapy. ${ }^{[1,3,4]}$ Thus, at initial management, the use of single-agent chemotherapy (MTX or ActD) should be the gold standard, 


\begin{tabular}{|c|c|c|}
\hline Variable & $\begin{array}{l}\text { Complete } \\
\text { response }\end{array}$ & $\begin{array}{l}\text { Failed } \\
\text { response }\end{array}$ \\
\hline Patients, $n(\%)$ & 34 (91.9\%) & $3(8.1 \%)$ \\
\hline Median age (years) (range) & $34(23-52)$ & $4 I(22-50)$ \\
\hline $\begin{array}{l}\text { Median World Health Organization } \\
\text { (WHO) prognostic score (range) }\end{array}$ & $3(0-6)$ & $5(3-5)$ \\
\hline Median duration of disease (months) & $I(I-10)$ & $3.5(1.5-11)$ \\
\hline
\end{tabular}

and combination therapy is not necessary and should be avoided. ${ }^{[6]}$ Primary combination therapy may be used in patients who are at an increased risk of resistance to single-agent chemotherapy-for example, patients with WHO prognostic scores of 5-6. ${ }^{[12]}$ Additional studies are required to better characterize which subgroups of low-risk patients may specifically profit from primary combination therapy. Lastly, patients who failed a sequential second-line single-agent chemotherapy may be considered for salvage combination therapy. ${ }^{[2]}$

Our hospital uses the 5-day IV MTX regimen, and the CR rate in our study was satisfactorily high $(n=34 / 37$, 91.9\%) with minimal toxicity profile. MTX is widely used in clinical practice because of its therapeutic, well-endured, and cost-effective qualities. It can be coadministered with or without FA to guard against the MTX-related toxicity. Globally, MTX is administered in diverse regimens-for example, weekly intramuscular (IM) MTX, 5-day IM MTX, 5-day IV MTX, 8-day IM MTX-FA, and high-dose intermittent infusion IV MTX-FA. ${ }^{[10]}$ To date, there is no worldwide agreement on the optimal regimen for MTX. However, the available data largely advocate that MTX regimens administered on 5- or 8-day schedules are the most effective ones. Most importantly, among all the MTX-based regimens, a recently published (2018) network meta-analysis concluded that the 5-day IV MTX regimen is the most effective schedule in producing $\mathrm{CR}$ in patients with low-risk GTN. ${ }^{[6]}$ In our study, we used the 5-day MTX regimen, which was not used by either of the two studies from Saudi Arabia. ${ }^{[2,7]}$ We found that our 5-day MTX regimen was highly effective in treating women with low-risk GTN, with CR rate of $92 \%$ and no severe adverse effects.

In our study, the CR rates to primary $(n=1)$ and sequential $(n=3)$ single-agent ActD chemotherapy were $100 \%$ and $100 \%$, respectively. ActD is a popular single-agent chemotherapy used in patients with low-risk GTN. ${ }^{[6]}$ It is most often exploited as a second-line single-agent therapy in patients with MTX resistance or when the use of MTX is contraindicated. ${ }^{[6,10,11]}$ In patients with resistance to MTX, sequential therapy with ActD has been shown to be associated with high CR rates nearly reaching $100 \%{ }^{[2,7]}$ Generally, the use of ActD is limited by its toxicity-related adverse events, such as hair loss, loss of appetite, diarrhea, and most significantly blister formation if extravasation takes place. The two most common regimens for single-agent ActD are 5-day IV ActD or pulsed IV ActD biweekly. ${ }^{[6,10,11]}$

According to a network meta-analysis of several randomized clinical trials and retrospective studies, ActD-based regimens were shown to be superior to MTX-based regimens in patients with low-risk GTN, particularly the pulsed IV ActD biweekly schedule. ${ }^{[6]}$ For that reason, some researchers currently advocate for the use of single-agent ActD in the initial treatment setting. In our hospital, there are ongoing discussions regarding the use of ActD as the first-line single-agent chemotherapy in patients with low-risk GTN. Until then, the hospital protocol will be 5-day IV MTX regimen in patients with the low-risk GTN.

From Saudi Arabia, to the best of our knowledge, only two studies have reported their experiences in the management of patients with low-risk GTN. ${ }^{[2,7]}$ In 2014, Anfinan et al. ${ }^{[7]}$ examined the treatment outcomes of low-risk GTN in 48 patients from King Abdulaziz University Hospital, Jeddah, Saudi Arabia. A total of 47 patients were treated with primary single-agent MTX chemotherapy, and 38 patients of them achieved CR of $81 \%$. On the contrary, the remaining one patient was treated with primary single-agent ActD chemotherapy and achieved CR of $100 \%$. All patients resistant to MTX chemotherapy $(n=11)$ were treated with salvage single-agent ActD, and all achieved CR of $100 \%$. MTX was administered in 8 -day regimen $(1 \mathrm{mg} / \mathrm{kg}$; days 1,3 , 5 , and 7$)$ alternating with FA $(0.1 \mathrm{mg} / \mathrm{kg}$; days $2,4,6$, and 8). ActD was administered in $1.25 \mathrm{mg} / \mathrm{m}^{2} \mathrm{IV}$ every 2 weeks. The OS for patients with low-risk GTN was $96 \%$.

In 2015, Al-Husaini et al. ${ }^{[2]}$ explored the treatment outcomes of low-risk GTN in 131 patients from King Faisal Specialist Hospital and Research Centre, Riyadh, Saudi Arabia. A total of 73 patients were treated with primary single-agent MTX chemotherapy, and 39 patients of them achieved CR of $53 \%$. On the other hand, a total of 23 patients were treated with primary single-agent ActD chemotherapy, and 20 patients of them achieved CR of $87 \%$. The remaining patients $(n=35)$ received primary combination therapy (for various reasons), and 32 patients of them achieved CR of $91 \%$. Among a selected number of patients who were resistant to MTX chemotherapy $(n=11)$, salvage treatment with single-agent ActD achieved CR of $82 \%$ in 9 patients. MTX 
was administered in either $1 \mathrm{mg} / \mathrm{kg}$ IM weekly or an 8-day regimen of $1 \mathrm{~g} / \mathrm{kg}$ IM every other day for four doses. ActD was administered in either $1.25 \mathrm{mg} / \mathrm{m}^{2} \mathrm{IV}$ every other week or $0.5 \mathrm{mg}$ IV daily for 5 days. The OS for patients with low-risk GTN was $100 \%$.

In Saudi Arabia, the overall incidence of GTN cannot be defined with a high level of certainty. This is because clinicopathologic data concerning the frequency of pregnancy and consequent GTN events are not available. Therefore, it is recommended to establish a formal national GTD registry in the country. Yearly meetings should be arranged whereby health-care providers meet to discuss the recent advances in the therapeutics of GTN; one of which is the consideration of ActD as the first-line single-agent therapy in patients with low-risk GTN. Lastly, referral of patients with GTN to highly qualified centers is greatly advised for proper risk stratification and clinical management.

The limitations of our study include its retrospective design, short-term survival data, single-center experience, and inclusion of only low-risk disease. It should be noted that our center does treat high-risk disease patients, and our single-center experience in the management of high-risk GTN will be reported in a future stand-alone communication. Furthermore, the limitation of small sample size hindered the potential to run an in-depth statistical analysis. However, despite these limitations, our study contributes additional data to the scarce literature available on low-risk GTN in Saudi Arabia.

\section{CONCLUSION}

In our study, we performed a retrospective analysis of patients with low-risk GTN who were treated at our center with 5-day IV MTX protocol. We found that our MTX regimen was highly effective in treating women with low-risk GTN, with CR rate of $92 \%$ and no severe adverse effects. Primary and sequential single-agent ActD therapy appears to be very effective for low-risk GTN.

\section{Acknowledgement}

This research work should be credited to the Department of Obstetrics and Gynecology, King Fahad Medical City, Riyadh, Saudi Arabia.

\section{Financial support and sponsorship}

Nil.

\section{Conflicts of interest}

There are no conflicts of interest.

\section{REFERENCES}

1. Seckl MJ, Sebire NJ, Berkowitz RS. Gestational trophoblastic disease. Lancet 2010;376:717-29.

2. Al-Husaini H, Soudy H, Darwish A, Ahmed M, Eltigani A, Edesa W, et al. Gestational trophoblastic neoplasia: Treatment outcomes from a single institutional experience. Clin Transl Oncol 2015;17:409-15.

3. Soper JT. Gestational trophoblastic disease. Obstet Gynecol 2006;108:176-87.

4. Berkowitz RS, Goldstein DP. Current advances in the management of gestational trophoblastic disease. Gynecol Oncol 2013;128:3-5.

5. Berkowitz RS, Goldstein DP. Current management of gestational trophoblastic diseases. Gynecol Oncol 2009;112:654-62.

6. Li J, Li S, Yu H, Wang J, Xu C, Lu X. The efficacy and safety of firstline single-agent chemotherapy regimens in low-risk gestational trophoblastic neoplasia: A network meta-analysis. Gynecol Oncol 2018;148:247-53.

7. Anfinan N, Sait K, Sait H. Gestational trophoblastic disease in the western region of Saudi Arabia (single-institute experience). Eur J Obstet Gynecol Reprod Biol 2014;180:8-11.

8. Golfier F, Labrousse C, Frappart L, Mathian B, Guastalla JP, Trillet-Lenoir V, et al. [Evaluation of treatment relating to gestational trophoblastic tumor registered to the French trophoblastic disease reference center (TDRC) in Lyon from 1999 to 2005]. Gynecol Obstet Fertil 2007;35:205-15.

9. McNeish IA, Strickland S, Holden L, Rustin GJ, Foskett M, Seckl MJ, et al. Low-risk persistent gestational trophoblastic disease: Outcome after initial treatment with low-dose methotrexate and folinic acid from 1992 to 2000. J Clin Oncol 2002;20:1838-44.

10. Lawrie TA, Alazzam M, Tidy J, Hancock BW, Osborne R. First-line chemotherapy in low-risk gestational trophoblastic neoplasia. Cochrane Database Syst Rev 2016;6:CD007102.

11. Alazzam M, Tidy J, Osborne R, Coleman R, Hancock BW, Lawrie TA. Chemotherapy for resistant or recurrent gestational trophoblastic neoplasia. Cochrane Database Syst Rev 2012;12:CD008891.

12. Sita-Lumsden A, Short D, Lindsay I, Sebire NJ, Adjogatse D, Seckl $\mathrm{MJ}$, et al. Treatment outcomes for 618 women with gestational trophoblastic tumours following a molar pregnancy at the Charing Cross Hospital, 2000-2009. Br J Cancer 2012;107:1810-4. 